\title{
Discussion on Performance Sense Training in Vocal Music Teaching Hongxing Ren
}

\author{
Music School, Shaanxi Normal University, Xi'an, 710119, China
}

\author{
Keywords: vocal music, performance, teaching
}

\begin{abstract}
Performance sense has always been one of the extremely important part in the performance skills of vocal music works. In the teaching of vocal music performance, it is required to practically change the situation of only paying attention to the training of vocalization skill but ignoring the training of performance sense. It is needed to actively guide the students learning vocal music performance to emphasize the performance sense cultivation and training in the process of performance and continuously improve the teaching method to continuously improve the performance sense of students in the vocal music singing and master the accuracy. Through the interpretation to the performance sense in the vocal music singing skills, the urgency of strengthening cultivation of performance sense is demonstrated in the training of vocal music singing skills, and several methods for strengthening performance sense training in vocal music singing skill are proposed.

Vocal music, as a kind of performance art, mainly means the re-composition activity by the performer after the first-stage creation of composer. The signing and performance of vocal music are restricted by the style and performance topic of songs and other various objective factors. In the process of performance, the accurate style mastering and good performance will assign the vocal music performance with rich expression and fresh vitality. In the process of performance, the performers must analyze the works performed meticulously and in detail, accurately master the style characteristics of the works, so as to master the performance sense in the process of singing and make the singing process become an artistic expression with lively organic combination of signing and performance, only in this way can the spiritual connotation in the songs be expressed in an artistic form with expression, distinctive layer and feeling tense. As an important part in the singing skills of vocal music, performance sense is the comprehensive display of performers in body shape, facial expression, eye expression and other various skills. Although seeming to be simple, vocal music performance is a complicated coordination process of singing-related muscle group, but the simple vocalization skill is no way equal to the vocal music art, the simple vocalization lack of feeling expression and proper performance is pale, dull and lack of affection and vitality. In the process of vocal music singing and performance, the proper gesture, facial expression and eye expression should be organically combined with the good sound skill. Only in this way will it able to ensure the transmission of feeling with sound and pay attention to the feeling transmission with body language, facial expression and eye expression in the performance of vocal music singing. So this requires the performers to fully absorb the essence of traditional drama performance of China, pay attention to the combination of hands, eyes, body, techniques and movements etc. in training and apply these artistic forms in the training of performance sense teaching of vocal music singing skills, so as to display the deep understanding and perfect presentation of vocal music performers to the performance sense.
\end{abstract}

\section{Interpretation of performance sense in vocal music singing skill}

The performance sense in the process of vocal music singing mainly refers to the construction of reasonable musical scene and feeling experience to stimulate the performance feeling of the performers to the song works. How the perform carry out performance design when receiving a vocal music work? First, it is required to deeply analyze the structural line, specific style, performance topic and connotation of the musical work, and meanwhile, it is required to deeply master the application of tone and the individual treatment, analyze the level of feelings expressed in the works, emotional strength and force. The purpose of vocal music performance is to express 
the inner feeling of a music work vividly and lively with human sound. The expression of feeling in signing has always been the long-term and constant artistic pursuit of the Chinese vocal music performers. The sincere feeling has a highlighted position in the singing performance. The feeling expression and performance in singing should be dominated by the pursuit of truth, here the truth means true feeling, true beauty and true sincerity. "Truth" means that the feeling expression and performance should conform to the status, personality and situation of the figures and conform to the general logics and law, with appropriate exaggeration, it should not be hokey pokey, let alone striking an attitude. Like "true watching, true listening and true experience", the vocal music performers should find out the feeling tone from the process of music work creation, specifically reflected in the inner happiness, anger, sorrow and pleasure and in the whole performance. It is required to experience the true feelings through sincerity, so as to light the hot feeling sparkle in the inner heart of audiences and generate resonance with the signers.

The author feels that the performance sense in the process of vocal music singing and some significant characteristics in the drama art require the performers to have the ability of playing various different roles. The style of music work is the regulation of roles, which requires that the performers should have very strong adaptation, and must be familiar with and master various diversified cultures, life experiences and feeling base related with music. Only in this way can there be an accurate performance sense. The cultivation of the sense requires to deeply understand the music work, and then it is required to transform the experience obtained in the process of interpretation into lively music image practically.

The operation in the process of music performance mainly involves in the specific operation ability of the performers. The technical level, imagination and psychological technical ability mastered by the performers all should be exercised with technology, and this requires the performers to think and practice frequently, so as to find out the inner feeling of singing in the process of performance. As for how to cultivate the vocal music performance learners in performance sense, through the education and teaching practice in recent years, especially through the specific analysis on the teaching training of performance feeling in the vocal music singing, the author feels that the following three points should be ensured at least: first, comprehensive consolidate the performance foundation of the learners, so as to change the situation of only paying attention to singing skill training while ignoring the psychological training and training of inner performance quality. Second, practically strengthen the performance training on the basis of education teaching. Third, devote to expanding the performance sense creation strategy of performers, apply various similar situations to stimulate the performance feeling of the performers. In this way, it is able to make the previous single and dull performance sense become fuller, so as to achieve the ultimate goal of improving the learners' performance ability,.

\section{Urgency to strengthen performance sense training in vocal music singing skills}

Literally, sense is completely a psychological term, but the sense in vocal music performance skill means the instinct reaction of organs felt by the human brains when facing various objective stimulators. After contacting the vocal music work, the performers should deeply and comprehensively analyze the structural line, music style and feeling connotation, and this is the premise to accurately master the performance sense in the process of vocal music singing. Thee mastering to the performance sense in the process of vocal music performance should also be able to make performers strongly resonate to the feelings expressed by the music work by using of feelings, just like the saying "to move others, you should move yourself first". However, in the actual teaching, many students are too weak in the foundation of sound skill, so they will spend a lot of energy and time in training skills. Because of this, the common method in vocal music class is to distribute $90 \%$ of the time to the sound skill training. But this necessary method make the students gradually enter the practice of only paying attention to the vocal skills, while ignoring the feeling expression of the work, let alone the stage performance with eye expression, feelings and gestures, to the extent tat the performance of vocal music students more and more tend to "blind learning", with a lack of feeling and performance. The direct result of this situation is that the vocal music 
students, whether undergraduate or postgraduate students, have fewer and fewer audiences in their concerts. Let alone the students of other majors, the number of student audiences of music major is also reduced sharply, and the situation that it is difficult to find a seat in the concert has gone. Although the situation cannot be described as only a few visitors, such phenomenon of serious loss of audiences should be sufficiently concerned by us. We should carefully rethink what problems have occurred in our vocal music performance, and what shortcomings exist in the class teaching. However, in the development history of vocal music, the vocal music artists making great success must be good at singing and performance. In the vocal music stage of China, among the old generation of singers, especially the female signers, many are good at singing and performance. They have left an indelible impression in the audiences with the stage image they create and depict. Their performance experience is still loved and recognized by the audiences even after decades of examination. It can be said that their success in art is inseparable from their excellent performance displayed on the stage. In the world opera stage, the performance masters well known in the world are all popular and pursued widely with the role they play and the figures they shape. When evaluating these great artists, people would say that the role shaped by a signer is so great and impressive, and they will never say that the invoice of someone is too pleasant simply. Those left in the mind of the audiences are more the stage artistic images shaped by the artists, but not only their beautiful singing sound. Based on this basic knowledge, if we ignore the training of performance training while only pay attention to the training of vocalization skills, although it cannot be said to run after the less important things, forgetting the important, for art, it is always imperfect and incomplete.

\section{Several countermeasures for strengthening performance sense training in vocal music signing skills}

(I) Build the awareness of equal importance of singing and performance

The good vocalization skill is very important, because sound is the carrier to transmit feelings and express meanings in the process of singing. Proper and appropriate body performance will add charm to the singing, making the performance contents more accurate and vivid, so it is easier to draw the attention of audiences and generate their feeling resonance. In the teaching and training of vocal music singing, it is required to special train the singing method of the learners, but also train their performance quality. Only having the essential singing quality can the learners perform freely when facing the content and form requirements of many different types of vocal music works. The vocal music course teachers must blend the training of performance ability into the overall training of vocal music performance.

(II) Strengthen special training on performance sense in the vocal music performance skills

1. Blend the ability cultivation and training of artistic scene

No performer can experience and feel the feeling experience of all artistic works, and this makes reference become an important learning and accumulation means in the process of performance. Performers should watch more performances, more performance videos, accumulate rich performance experience and performance materials. For the experience and feeling of various emotions, it is also required to take advantage of the literature works and TV \&film works, in these artistic forms, various feelings and emotions can provide us with rich emotional experience.

2. Body shape, movement and eye expression training

The stage performance is inseparable from the gesture movement. The training for the gesture performance movement must be blended into the class. The class is the stage, and the performers must build the awareness and ability of taking singing as performance. The gesture movement and vocabulary must be trained in a targeted manner, the performers can systematically learn and train the basic movement and principle of the performance by seeking help from the dance teacher and drama performance teachers. Works of different styles must be expressed and performed in different forms. For the different emotions like happiness, warmness, passion, joy, pleasure, sadness, sorrow, worry and despair, there must be corresponding body gestures, facial expressions and eye expressions. 
(III) Strengthen the incentives of learners for creation of vocal music performance creation

It is required to strengthen the vocal music students' subject awareness in singing and performance and the incentives in artistic creation. This is because the traditional vocal music teaching is mainly to interpret the vocal music works through teachers, and then ask the learners to train their singing ability in classroom. However, generally, it is difficult to mobilize the incentive and performance awareness of the performers in the process of vocal music singing. In order to further promote the learners to actively experience and master the performance feelings in vocal music singing, teachers can adjust the teaching method in the process of vocal music class teaching and expand the students' performance and creation ability continuously. Teachers can adopt many methods for active guidance, so that the students can fully expand their imagination to understand and master the idea and theme of the vocal music works, actively feel the emotion and feeling of the vocal music works in the form of imagination and empathy, find out their own performance thought well in the process of performance scene they create, enrich their performance experience and cultivate the performance sense. The students are allowed to make mistake and have their shortcomings in understanding and performance accepted, so as to gradually build the students' subject awareness and subjectivity, in this way, the students can deeply experience the inner meaning of the music work gradually, specifically and consciously, so as to achieve active awareness in signing and performance, gradually achieve the seamless joint between signing skills and performance skills and performance sense, blend singing and performance in complete harmony and make the artistic image full and perfect.

\section{Conclusion}

Overall, only by continuously strengthening the training of performance feeling in the vocal music singing skill can the learners comprehensively and deeply experience the subject of vocal music with rich culture and feeling connotation. To control vocal music art skillfully and perform successfully, it is not only required to master the good vocalization skill, but also deeply learn various artistic cultures to enrich the learners themselves, so as to own richer and more extensive knowledge, quality and emotional experience accumulation, only in this way can the learners have good performance sense in signing performance, shape successful stage image, attract and move more audiences. The realistic life is rich and colorful, as long as the learners are good at observing and experiencing life, learning from relevant artistic forms and continuously absorbing the nutrition, the performance ability of work must be increased inevitably. In the process of signing, the learners should fully play their artistic imagination, comprehensively blend into the feeling world of works and achieve the perfect combination of "singing" and "performance", only in this way can be vocal music performance have more affection and vitality. Only by comprehensively dealing with the relation between signing and performance will it be able to create a more perfect aesthetic space, win the welcome, recognition and praise of more audiences.

\section{References}

[1] Yang Yihe, Principle and Application of Music Performance Art [M], Anhui Literature and Art Publishing House, 2002

[2] Zhu Qi. Brief Discussion on Relevant Issues of Emotion and Psychology in Vocal Music [J] Journal of Taiyuan Institute of Education, 2005 (2)

[3] Wang Shanhu, On Feeling and Thinking in Vocal Music Performance [D], Wuhan Conservatory of Music, 2008

[4] Sun Rujie, On Importance of Theoretical Research and Performance Practice in Vocal Music Singing-Thinking from Vocal Music Teaching Practice [J], Music Life, 2014 (1) 